children aged 9-24 mo from a large urban area of Argentina. Am J Clin Nutr. 1990; 52(3):534-40.

7. O’Donnell A M, Carmuega E, Durán P. Preventing Iron Deficiency in Infants and Preschool Children in Argentina. Nutr Rev. 1997; 55(6):189-94.

8. Morasso M del C, Molero J, Vinocur P, Acosta L, et al. Deficiencias de hierro y de vitamina A y prevalencia de anemia en niños y niñas de 6 a 24 meses de edad en Chaco, Argentina. Arch Latinoam Nutr. 2003; 53(1):21-7.

9. Morasso M del C, Molero J, Vinocur P, Acosta L, et al. Deficiencia de hierro y anemia en mujeres embarazadas en Chaco, Argentina. Arch Latinoam Nutr. 2002; 52(4): 336-43.

10. Varea A, Malpeli A, Etchegoyen G, Vojkovic M, et al. Short-term evaluation of the impact of a food program on the micronutrient nutritional status of Argentinean children under the age of six. Biol Trace Elem Res. 2011; 143(3):1337-48.

11. Malpeli A, Ferrari MG, Varea A, Falivene M, et al. Shortterm evaluation of the impact of a fortified food aid program on the micronutrient nutritional status of Argentinian pregnant women. Biol Trace Elem Res. 2013; 155(2):176-83.

12. Calvo E, Biglieri A. Impacto de la fortificación con ácido fólico sobre el estado nutricional en mujeres y la prevalencia de defectos del tubo neural. Arch Argent Pediatr. 2008; 106(6):492-8.

13. Bernztein R, Drake I. Subprescripción de hierro y variabilidad en el primer nivel de atención público de la Argentina. Arch Argent Pediatr. 2008; 106(4):320-7.

14. Ceriani Cernadas JM, Carroli G, Pellegrini L, Ferreira M, et al. Efecto del clampeo demorado del cordón umbilical en la ferritina sérica a los seis meses de vida: Estudio clínico controlado aleatorizado Arch Argent Pediatr. 2010; 108(3):201-8.

15. Marin GH, Mestorino N, Errecalde J, Huber B, et al. Personalised iron supply for prophylaxis and treatment of pregnant women as a way to ensure normal iron levels in their breast milk. J Med Life. 2012; 5(1):29-32.

16. WHO, UNICEF. Global Strategy on infant and young child feeding. 2012. [Acceso: 21 de febrero de 2020]. Disponible en: https: / / www.who.int/nutrition/ publications / infantfeeding/9241562218/en/

17. Neville MC, Anderson SM, McManaman JL, Badger $\mathrm{TM}$, et al. Lactation and neonatal nutrition: defining the critical questions. J Mammary Gland Biol Neoplasia. 2012; 17(2):167-88.

18. Mozaffarian D, Rosenberg I, Uauy R. History of modern nutrition science-implications for current research, dietary guidelines, and food policy. BMJ. 2018; 361:k2392.

\title{
Estudio clínico-epidemiológico de las infecciones respiratorias agudas bajas causadas por adenovirus en niños hospitalizados. Diecinueve años de vigilancia epidemiológica activa [Comentario]
} Clinical and epidemiological study of acute lower respiratory tract infections caused by adenovirus in hospitalized children. Nineteen years of active epidemiological surveillance [Commentary]

Las infecciones respiratorias agudas son la principal causa de morbilidad en la población pediátrica. Si bien en las áreas de bajos recursos la carga de enfermedad es mayor, en las áreas de mayores recursos los desafíos son la resistencia a los antibióticos en las infecciones bacterianas y la identificación de nuevos patógenos. ${ }^{1,2}$

En los últimos años se fue generando un creciente desarrollo en la identificación de virus respiratorios y el rol que presentan en las neumonías de la población pediátrica. Con las técnicas moleculares de diagnóstico virológico se informa la detección de hasta un $92 \%$ de virus respiratorios en niños con neumonía. Los virus asociados a neumonía de la comunidad son el adenovirus humano (AdVH), bocavirus humano $(\mathrm{BoVH})$, coronavirus humano $(\mathrm{CoVH})$, enterovirus (EV), virus de la gripe (Flu), metapneumovirus (hMPV), virus parainfluenza (VPI) y virus sincicial respiratorio (VSR). ${ }^{3,4}$
En el estudio de casos y controles realizado por Rhedin y col. en Estocolmo en el que analizaron la asociación de virus respiratorios y neumonía en menores de 5 años, se detectaron virus en 98/121 (81\%) niños con neumonía y 134/ 240 (56 \%) de los controles. En el análisis univariado los virus asociados significativamente con neumonía de la comunidad fueron hMPV $(p<0,001)$, VSR $(p<0,001)$, Flu $(p=0,03)$ y $\operatorname{AdVH}(p=0,05)$, mientras que en el análisis multivariado AdVH no se asoció significativamente con neumonía y se detectó con bastante frecuencia en los controles. ${ }^{3}$ Esto podría relacionarse a que, si bien se sabe que el adenovirus es un patógeno que causa neumonía de la comunidad, se ha observado que puede persistir en el tejido linfático amigdalino durante semanas. Por otra parte también se ha descrito la latencia del adenovirus en los pulmones y la reactivación en el huésped inmunosuprimido. ${ }^{5}$ 
Nacimiento-Carvalho y col., detectaron virus en $708(91,5 \%$; IC $95 \%: 89,3-93,3)$ casos de neumonía en menores de 5 años, de los cuales en 491 (69,4\%; IC $95 \%$ : 65,9-72,7) se identificó coinfección viral. Rinovirus (46,1\%; IC $95 \%$ : 42,6$49,6)$, adenovirus $(38,4 \%$; IC $95 \%: 35,0-41,8)$ y enterovirus $(26,5 \%$; IC $95 \%$ : 23,5-29,7) fueron los virus más comúnmente detectados y la combinación hallada más frecuentemente fue rinovirus con adenovirus. ${ }^{4}$

Si bien el adenovirus fue el virus respiratorio identificado con menor frecuencia durante todo el período estudiado por Bakir y col., en el artículo que se publica en este número de Archivos, hallándose en el 4,4 \% (265/6047) del total de los casos positivos, esto podría relacionarse con la menor sensibilidad y especificidad de la IFI. Por otra parte, la inclusión de niños con bronquiolitis también podría haber contribuido a una menor proporción en la detección. Como se refiere en el artículo, la presentación más frecuente fue neumonía, con una asociación significativa.

En un estudio, Yu Lin y col., demostraron una mayor frecuencia de virus respiratorios en población pediátrica con RT-PCR $(75,3 \%)$ que con las pruebas tradicionales $(49,3 \%)$. Los principales patógenos detectados fueron VSR $(23,8 \%)$, rinovirus $(15,2 \%)$, PIV3 (11,2\%), FluA $(10,8 \%)$ y $\operatorname{AdVH}(10,1 \%)$. Para los niños menores de 5 años, el VSR y rinovirus fueron los más frecuentes, mientras que en los niños mayores de 5 años fueron FluA y AdVH. ${ }^{7}$

Por otra parte, en Bakir, ${ }^{6}$ es importante, entre los factores asociados, la identificación de un alto porcentaje de niños con comorbilidades, posiblemente relacionado a las características de la institución. Esto también puede observarse en la letalidad, en donde de los 20 niños que fallecieron el $55 \%$ tenía alguna comorbilidad.

Como predictores independientes de la infección por adenovirus se identificaron el sexo masculino, la edad igual o mayor de 12 meses, la reinternación, la internación previa por causas respiratorias y la presentación clínica de neumonía, lo cual brinda una importante información para la sospecha de este agente etiológico.

Además, considerando que uno de los predictores fue el diagnóstico de ingreso de neumonía, es importante reforzar la importancia de la detección de virus respiratorios en las infecciones del tracto respiratorio inferior para establecer una conducta adecuada con el tratamiento y la necesidad de aislamiento. Tal como se menciona en el estudio de Bakir, los métodos de biología molecular permiten una mayor frecuencia en la identificación de los virus respiratorios y la IFI es una técnica adecuada para el tamizaje.

Es importante continuar con la vigilancia epidemiológica y el análisis de la información, para identificar los cambios en el tiempo que podrían presentarse con la incorporación de la vacunación antineumocóccica y una adecuada cobertura. De esta manera, se facilita el uso adecuado de los antibióticos y las medidas de prevención para disminuir la transmisión dentro de las instituciones de salud.

Dra. Miriam Bruno

División de Promoción y Protección de la Salud Hospital Carlos G. Durand. CABA

http:/ / dx.doi.org/10.5546/ aap.2020.158

Texto completo en inglés:

http:/ / dx.doi.org/10.5546/aap.2020.eng.158

Cómo citar: Bruno M. Estudio clínico-epidemiológico de las infecciones respiratorias agudas bajas causadas por adenovirus en niños hospitalizados. Diecinueve años de vigilancia epidemiológica activa. [Comentario]. Arch Argent Pediatr 2020;118(3):158-159.

\section{REFERENCIAS}

1. Liu L, Oza S, Hogan D, Perin J, et al. Global, regional, and national causes of child mortality in 2000-13, with projections to inform post-2015 priorities: An updated systematic analysis. Lancet. 2015; 385(9966):430-40.

2. Walker CL, Rudan I, Liu L, Nair H, et al. Global burden of childhood pneumonia and diarrhoea. Lancet. 2013; 381(9875):1405-16.

3. Rhedin S, Lindstrand A, Hjelmgren A, Ryd-Rinder M, et al. Respiratory viruses associated with community-acquired pneumonia in children: matched case-control study. Thorax. 2015; 70(9):847-53.

4. Nascimento-Carvalho AC, Vilas-Boas AL, Fontoura MH, Vuorinen T, et al. Respiratory viruses among children with non-severe community-acquired pneumonia: A prospective cohort study. J Clin Virol. 2018; 105:77-83.

5. Kalu SU, Loeffelholz M, Beck E, Patel JA, et al. Persistence of adenovirus nucleic acids in nasopharyngeal secretions: a diagnostic conundrum. Pediatr Infect Dis J. 2010; 29(8):746-50.

6. Bakir J, Juárez MV, Lución MF, Areso MS, et al. Estudio clínico-epidemiológico delasinfecciones respiratorias agudas bajas causadas por adenovirus en niños hospitalizados. Diecinueve años de vigilancia epidemiológica activa. Arch Argent Pediatr 2020;118(3):193-201.

7. Lin CY, Hwang D, Chiu NC, Weng LC et al. Increased Detection of Viruses in Children with Respiratory Tract Infection Using PCR. Int J Environ Res Public Health. 2020; 17(2):E564. 